\title{
SCENE AND OBJECT CLASSIFICATION USING BRAIN WAVES SIGNAL
}

\author{
DARSHAN A KHADE*, ILAKIYASELVAN N* \\ Department of Computing Science and Engineering, VIT University, Chennai Campus, Tamil Nadu, India. Email: darshanashokrao. \\ khade2015@vit.ac.in/ilakiyaselvan.n@vit.ac.in
}

Received: 23 January 2017, Revised and Accepted: 03 March 2017

\begin{abstract}
This study aims to classify the scene and object using brain waves signal. The dataset captured by the electroencephalograph (EEG) device by placing the electrodes on scalp to measure brain signals are used. Using captured EEG dataset, classifying the scene and object by decoding the changes in the EEG signals. In this study, independent component analysis, event-related potentials, and grand mean are used to analyze the signal. Machine learning algorithms such as decision tree, random forest, and support vector machine are used to classify the data. This technique is useful in forensic as well as in artificial intelligence for developing future technology.
\end{abstract}

Keywords: Support vector machine, Electroencephalograph, Independent component analysis.

(c) 2017 The Authors. Published by Innovare Academic Sciences Pvt Ltd. This is an open access article under the CC BY license (http://creativecommons. org/licenses/by/4. 0/) DOI: http://dx.doi.org/10.22159/ajpcr.2017.v10s1.19594

\section{INTRODUCTION}

In today's world, technology is developing very rapidly day by day; on the other side, crime also increases. To tackle this problem, Hans Berger's invented the electroencephalograph (EEG) machine which is used to record the electrical activities happen inside brain within the scalp of the human [1]. These EEG signals are very useful in artificial intelligence, medical as well as in forensic laboratory. Scene and object classification are useful in artificial intelligence and forensic laboratory. In scene classification, I have taken the dataset of the individual on whose scalp we placed the electrode for capturing the signals, and then I show moving squares on the screen as object. The scene is accepted to the individual; seeing these objects, EEG signals are acquired and named as square as reaction of individual. This analysis is useful in many aspects such as if any person is suspect in some crime activity like murder. Then, we can tell whether the suspect knows about the crime or not by analyzing the suspect's brain waves signals. If he/she knows or if he/she present at the moment of crime, his/her brain signals differ from normal signals [2]. Fig. 1 shows the process of analyzing the signals. First, we need to filter the signal from the dataset; when any person views any scene or object, their eyes and memory works thus filtered signals get only signals from Frontal and Occipital which helps to get desired signal for analysis for prediction.

\section{LITERATURE SURVEY}

To capture the electrical activities inside the brain, EEG device is used which helps us to record the brain waves in the form of EEG signals. There are various types of neuroheadsets which help to capture this signals described by Teplan [1] of different kinds of brain waves as delta, theta, alpha which help to detect the condition of the human brains [3]. Fosu and Jouny described that our brain signal are also able to response the different colors and the brain signals differ from each other. They may be stationary or non-stationary signals $[4,5]$. It is also possible that we can detect the shapes of the objects seen by individual, by analyzing their brain waves signal and also by using the tactile signal which produces by touch perception described by Khasnobish et al. [7]. Brain-computer interface is used to provide an interface between the human brain and the computer which is used in artificial intelligence for developing the human body-related devices which work on human brain. We can classify the dataset using the various machine learning algorithms which help to classify the data described by Anupama et al., [6].
Similar to EEG signal using Tactile signal also, we can determine the shape of objects using sensitivity signal of the body [8]. To extract the required features from EEG signals, various feature extraction techniques such as fast Fourier transform and Wavelet transformation (WT) are used [9]. By using independent component analysis (ICA), we can separate the required signals from other signals which helps to get separate signals as per channels [10]. Several noise and artifact reduction filters are available which help to reduce the noise from the acquired EEG signals. Machine learning algorithms are useful to predict and classify the data by supervised and unsupervised algorithms such as classification and clustering methods. EEG signal analysis is also very useful in the forensic laboratory for detecting the lie; when any individual lies, their signals differ as compared to normal signal which helps to predict and thus it is also used as lie detector described by Amir et al., [2].

\section{METHODS}

\section{Preprocessing}

The acquired EEG signal is filtered to remove linear trends using linear finite impulse response filtering. To get the desire signal, the signal is rereferenced from fixed or common reference to average reference. After extracting the epochs, a reference point was used to check with time. Interval from the filtered dataset. Moreover, removed the baseline and arising from low-frequency artifacts to form the dataset. Fig. 2 shows the signal waves with respect to the channel numbers that we chose after filtering the data so that we can reduce the work by removing the noisy data and selecting the signals which are required.

\section{Feature extraction}

Fourier transform is one of the extracting methods for EEG signals [9]. EEG signal is non-stationary; the most suited feature extraction from the raw data is to apply the time-frequency domain method like WT feature extraction technique can be expressed as an infinite series of wavelets. The original EEG signals are represented as secured and simple blocks known as wavelets. A big or mother wavelet gives rise to these wavelets as part of derived functions through translation and dilation on shifting, compression, and stretching operations on the time axis.

\section{ICA}

Due to many disturbances, unwanted signals such as movement of eyes and breathing get recorded. To improve the result, we choose to remove the "unwanted noise" from signal using ICA [10]. This technique is 


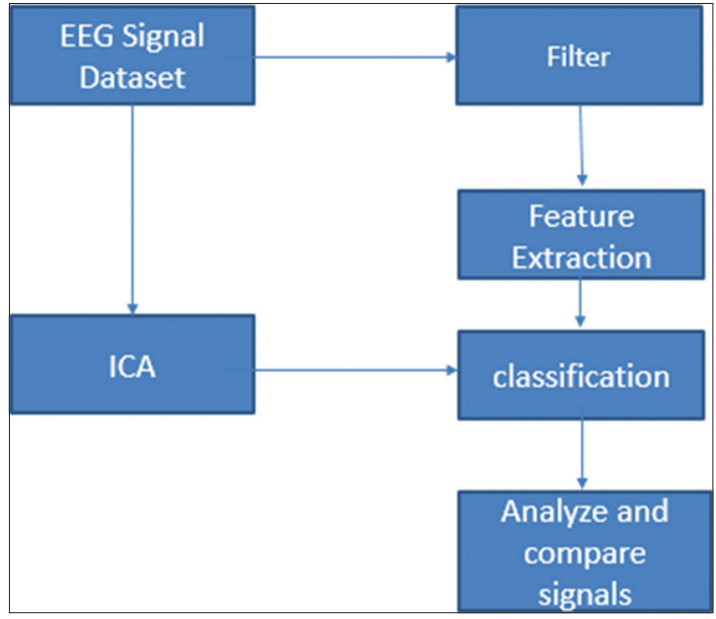

Fig. 1: Block diagram of signal analysis

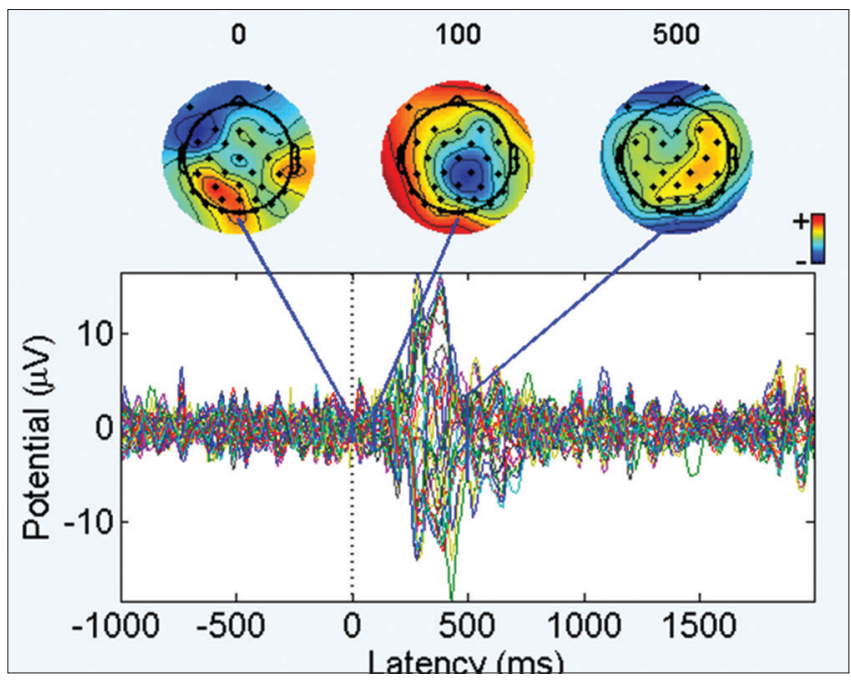

Fig. 2: Scalp image

useful to produce good result of reduction in noisy data from EEG signals. The independent component filters are used to separate signals from multivariate signals available in the channel data.

\section{Classification}

Machine learning algorithms are used to classify the signal data and provide efficient result [7]. Decision tree (DT) is one of the supervised algorithms which is used to classify the data in a tree structure manner. Continuous signals are decided as splitting measure for the branches in DT. Similar to DT, random forest is more advance classification algorithm which generates many trees; on the basis of majority output, result will be decided. It is more precise than DT algorithm; another classification algorithm is support vector machine used to arrange the data as it is derived from statistical learning theory. It uses the hypothesis space of linear function [11]. It classifies the data using the hyperplane by finding the Euclidean distance between the vector points and hyperplane; the line which has the maximum distance from vector point is considered as hyperplane.

\section{RESULT}

This study is carried to check the object shape detection using EEG signal and filtered reference. The data divided into two parts and compared together to find the grand mean which provides more accurate data. ICA used to separate signals from multivariate signals and reduce the noisy data using the feature extraction and its algorithms. By setting event-related potentials (ERP) signals of each

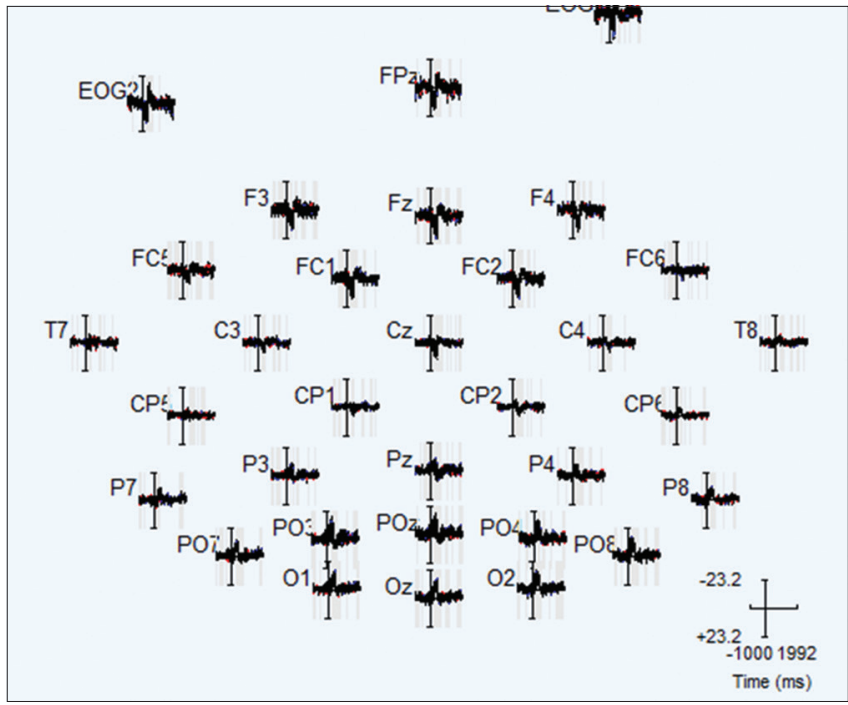

Fig. 3: Event-related potentials signals per channel location

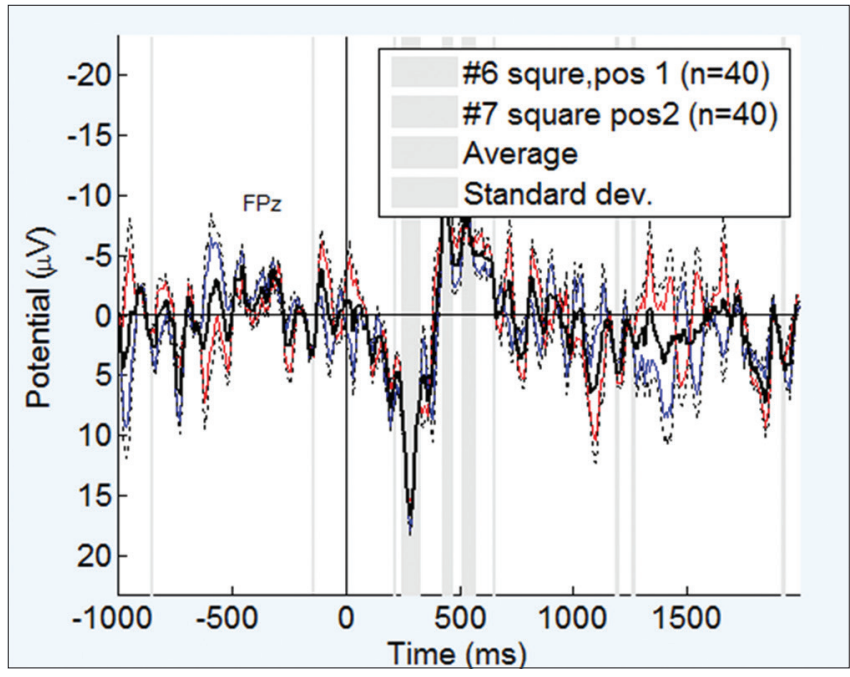

Fig. 4: Comparing event-related potentials in two conditions

channel with their mean values, we can see the changes according to the object changes.

Fig. 3 shows the analysis of signals. Fig. 3 shows the ERP signal per channel and Fig. 4 shows the signal compared the mean of a particular channel. In future, we can compare other signals with unknown scene and object so that we can classify the known and unknown events.

\section{CONCLUSION AND FUTURE WORK}

This paper aims to analyze the EEG signal of an individual which has shown the known objects. First, filtered the data and then extracted the required signal using features extraction so that noisy data get removed to perform the grand mean operation on it so that we can analyze the signal. ICA is performed to separate signal from multivariate signals.

In future, we will record the two different EEG signal dataset with known and unknown scene objects and then analyze and compare the signals so that we can classify the scene and objects using brain waves signals.

\section{REFERENCES}

1. Teplan M. Fundamentals of EEG measurement. Meas Sci Rev 2002;2:1-11 
2. Amir S, Ahmed N, Chowdhry BS. Detection in Interrogations Using Digital Signal Processing of Brain Waves 2013. Bandung: $3^{\text {rd }}$ International Conference (ICICI-BME), November 7-8; 2013.

3. Zhu G, Li Y, Wen PP. Analysis and classification of sleep stages based on difference visibility graphs from a single-channel EEG signal. IEEE J Biomed Health Inform 2014;18:1813-21.

4. Fosu KP, Jouny I. Separation and classification of EEG responses to color stimuli (NEBEC), 2015. 41 ${ }^{\text {st }}$ Annual Northeast Year; 2015. DOI: 10.1109/NEBEC.2015.7117185.

5. Zhang H, Tang Z. To judge what color the subject watched by color effect on brain activity. IJCSNS Int J Comput Sci Netw Secur 2011;11:80-3.

6. Anupama HS, Cauvery NK, Lingaraju GM. Real-time EEG Based Object Recognition System Using Brain Computer Interface. International Conference on Contemporary Computing and Informatics (IC3I); 2014.

7. Khasnobish A, Jati A, Bhattacharyya S, Konar A, Tibarewala DN,
Janarthanan R, et al. Object-Shape Classification and Reconstruction from Tactile Images Using Image Gradient. IEEE $3^{\text {rd }}$ International Conference. Kolkata, India: Emerging Applications of Information Technology; 2012. p. 93-6

8. Datta S, Pal M, Khasnobish A, Tibarewala DN, Janarthanan R. Correlation Analysis of Object Shape Recognition from EEG and Tactile Signals. Advances in Electrical Engineering (ICAEE); 2014. DOI: 10.1109/ICAEE.2014.6838444.

9. Karpagachelvi S, Arthanari M, Sivakumar M. ECG Feature Extraction Techniques - A Survey Approach. No. 1. Vol. 8. USA: (IJCSIS) International Journal of Computer Science and Information Security; 2010 .

10. Liu Y, Beadle PJ. Independent Component Analysis of EEG signals; 2005. p. 219, 222. 28-30 May. DOI: 10.1109/IWVDVT.2005.

11. Lal TN. Support vector channel selection in BCI. IEEE Trans Biomed Eng 2004;51:1003-10. 